\title{
DEGRADATION OF NATURAL ORGANIC MATTER FROM SURFACE WATER USING SONOLYSIS
}

Mihai Stefanescu, Cristiana Cosma, Ionut Cristea, loana Ionescu, Costel Bumbac

National Research and Development Institute for Industrial Ecology ECOIND, 71-73

Drumul Podu Dambovitei Street, sector 6, Bucharest, Romania

tehnologi@incdecoind.ro,

\begin{abstract}
This paper shows the behavior of natural organic matter (NOM) from drinking water under the influence of ultrasonic radiation. Experimental tests were performed by direct sonolysis of a drinking water source (river water) in order to establish the influence of main parameters (water source composition, irradiation time, energy and amplitude of ultrasonic wave, temperature, $\mathrm{pH}$, conductivity) on NOM efficiency degradation emphasized by total organic carbon (TOC) and CODMn indicators.

Likewise, UV-VIS spectra were made for the characterization of degradation processes based on nonconventional parameters A254, A280, A365, SUVA.

The experimental results highlight maximum $30 \%$ efficiency of NOM removal for short irradiation time, two minutes, diminishing of the ultrasonic wave amplitude leading to removal efficiency increasing (for the same $16 \mathrm{kHz}$ frequency and the same energy of the wave).
\end{abstract}

Keywords: natural organic matter, NOM, potabilization, sonolyse, TOC

\section{Introduction}

Ultrasonic radiation is a vibration of an elastic medium (water, gases, solids) having over $16 \mathrm{kHz}$ frequency with many application in medicine, industry, biology as well as in water/wastewater treatment.

Ultrasonic waves passing through the water generate small bubbles of gas which grow and collapse very quickly (milliseconds) releasing large energy amounts but in small area of the cavities surroundings.

Hot spots theory is general accepted to explain physical and chemical processes occurring during ultrasonic irradiation

Since 1990s ultrasound has been widely used in water and wastewater treatment processes as an emerging advanced oxidation process (AOP) technology, applicable for a wide range of contaminants with various initial concentrations.

Generation, combination and recombination of free radicals, very reactive, able to degrade pollutants from water are the base of aqueous solutions sonochemistry.

The mechanism of micropollutant degradation during sonolysis can be described as two steps process. During the first step, $\mathrm{H}_{2} \mathrm{O}$ and $\mathrm{O}_{2}$ are sonolysed inside the cavitation buble and in the second step, $\mathrm{HO} \bullet$ and $\mathrm{HOO} \bullet$ radicals 
move to the liquid-bubble interface to react with the organic substrate or to recombine with each other to form $\mathrm{H}_{2} \mathrm{O}_{2}$.

Ultrasonic frequency, electric power of ultrasonic generator, type of transducer, reaction $\mathrm{pH}$, nature of pollutants, sonication time, additives (inorganic salts, acids or gases, solid particles) are some of the main factors which can control the degradation efficiency of pollutants [1].

Scientifically research in this field since ' 90 proved that direct sonolysis is efficient for advanced degradation of organic pollutants in water. Ultrasounds become more efficient in both pollutants degradation and economical if sonolyse is associated with other classical and AOPs methods for water treatment: US $+\mathrm{H}_{2} \mathrm{O}_{2}, \mathrm{US}+\mathrm{H}_{2} \mathrm{O}_{2}+\mathrm{Fe}, \mathrm{US}+\mathrm{O}_{3}, \mathrm{US}+\mathrm{UV}, \mathrm{US}+$ electrochemical oxidation, US + adsorption [2].

Natural organic matter (NOM) has a negative impact to drinking water quality and is precursor for trihalomethans and haloacetic compounds formation during the disinfection with chlorine, in drinking water treatment plants [3,4]. Disinfection with chlorine is a widespread method because is efficient, less expensive and assures the water quality at the end of pipe. For these reasons is preferred this disinfection method noting that must be taken measures for advanced remove of NOM in the potabilization flow and to keep clean, without breaches the drinking water distribution network [5].

Conventional and nonconventional indicators are used for the characterization of drinking water. Literature data mentioned some nonconventional parameters which are well known (i.e. SUVA) because these indicators are referring both to the composition of NOM (humic acids, fulvic acids, hydrophilic and hydrophobic compounds a.s.o.) and to reaction ability with coagulants and oxidants (especially with chlorine). All of them are based on data supplied by UV spectra, for different wavelengths of the polluted and treated water $[6,7]$

\section{Materials and Methods}

The aim of experimental tests was to demonstrate at laboratory level the efficiency of direct sonolysis for the degradation of natural organic matter (NOM).

Vibracell VCX500/Sonics generator having a standard probe (transducer) from titanium alloy supplied the ultrasonic field for water treatment (river water). 20 $\mathrm{kHz}$ was the single operating frequency for all experiments. It was tested different energies (555-2500 W) and amplitudes (25-100\%) of the ultrasonic waves.

Physical-chemical characterization of raw water and treated water was performed based on the following conventional and nonconventional indicators:

- conventional indicators: pH (SR EN ISO 10523:2012), turbidity (SR EN ISO 7027:2001), conductivity (SR EN 27888-97), COD-Mn (SR EN ISO 8467-01), DOC (SR EN 1484:2006), temperature;

- nonconventional indicators (UV spectrometry): A254 (absorbance of the aqueous sample to $\lambda=254 \mathrm{~nm}$ ), A280 (absorbance of the aqueous sample to $\lambda=280 \mathrm{~nm}$ ), A365 (absorbance of the aqueous sample to $\lambda=$ $365 \mathrm{~nm}$ ), SUVA (ratio A254/DOC), ratio A254/A280, ratio A254/A365. 
Two series of sonolyse tests were performed: sonolyse of raw water (in this case the wave amplitude was constant $100 \%$ ) and sonolyse of pretreated water - coagulation with aluminum sulphate $(10 \mathrm{mg} \mathrm{Al} / \mathrm{l}$, five minutes stirring at 100 rpm) and settling (30 minutes). The variable parameters for sonolysis experiments were as follows:

- wave energy $(555 \div 2500 \mathrm{~W})$;

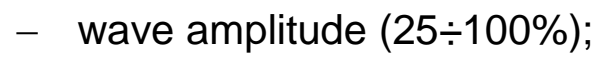

- irradiation time $(15 \div 600$ seconds).

Spectral analyses were performed in case of pretreated water tests in order to select nonconventional indicators for a better characterization of the samples.

\section{Results and Discussion}

\section{a) Sonolyse of raw water}

The experimental results emphasized the following aspects (table 1):

- in case of experiments carried out to high energy (2500 W), increasing irradiation time leads to higher values of turbidity (max. 17\%) because of large particles disintegration in contact with ultrasonic waves (samples A1-A4). The maximum turbidity values was 201 NTU (two minutes irradiation time). The temperature increased too with irradiation time from $23^{\circ} \mathrm{C}$ to $65^{\circ} \mathrm{C}$ after ten minutes of sonolysis, the highest value being in case of A5 sample.

A special mention for A5 sample (irradiation time $=10 \mathrm{~min}$.) that suggests the possible influence of high temperature $\left(65^{\circ} \mathrm{C}\right)$ upon solubilization phenomenon, emphasized by the decreasing of turbidity from 201 NTU (irradiation time $=2 \mathrm{~min}$.) to $159 \mathrm{NTU}$. Likewise conductivity increased with irradiation time from $468 \mu \mathrm{S} / \mathrm{cm}$ to $845 \mu \mathrm{S} / \mathrm{cm}$ showing that a part of organic and inorganic matter which was in suspension or in colloidal form is solubilized during cavitation process which generates high pressures and temperatures (hot spots). The global organic load expressed by COD-Mn and DOC decreased with $20 \%$ and $32 \%$ respectively in case of short irradiation time (15-30 sec.) but had begun to rise for longer irradiation time, surpassing even initial value $(2.88 \mathrm{mg} \mathrm{C} / \mathrm{l})$ in case of sample A5 (3.14 $\mathrm{mg}$ $\mathrm{C} / \mathrm{l}$ ) when the organic soluble substances were $9 \%$ higher than raw water;

- turbidity increased with $48 \%$ after two minutes of sonolyse (sample B4) in case of medium energy experimental tests $(1110 \mathrm{~W})$, temperature in the same way but conductivity increased more than $(250 \%)$ similarly samples at high energy for longer irradiation time: $1194 \mu \mathrm{S} / \mathrm{cm}$ (sample B5) indicating solubilization of solid phase. This phenomenon is accomplished by organic load (CODMn) increasing in time (after ten minutes with $80 \%$ ). 
Table 1. Sonolyse experiments of raw water (wave amplitude 100\%)

\begin{tabular}{|c|c|c|c|c|c|c|c|c|}
\hline Sample & $\begin{array}{c}\text { Energy, } \\
\text { W }\end{array}$ & $\begin{array}{l}\text { Irrad. } \\
\text { time } \\
\text { sec. }\end{array}$ & $\mathrm{pH}$ & $\begin{array}{c}\text { Turbidity } \\
\text { NTU }\end{array}$ & $\begin{array}{c}\text { Temp. } \\
{ }^{\circ} \mathrm{C}\end{array}$ & $\begin{array}{c}\text { Conductivity } \\
\mu \mathrm{S} / \mathrm{cm}\end{array}$ & $\begin{array}{c}\text { COD-Mn } \\
\mathrm{mg} \mathrm{O}_{2} / \mathrm{l}\end{array}$ & $\begin{array}{l}\mathrm{DOC} \\
\mathrm{mg} \mathrm{C} / \mathrm{l}\end{array}$ \\
\hline $\begin{array}{c}\text { Raw } \\
\text { water }\end{array}$ & - & - & 7.60 & 142 & 23 & 468 & 6 & 2.88 \\
\hline $\mathrm{A} 1$ & 2500 & 15 & 7.56 & 172 & 25 & 486 & 4.66 & 2.45 \\
\hline A2 & 2500 & 30 & 7.26 & 186 & 28 & 482 & 4.83 & 1.96 \\
\hline A3 & 2500 & 60 & 7.30 & 188 & 32 & 522 & 7.00 & 2.20 \\
\hline A4 & 2500 & 120 & 7.37 & 201 & 40 & 589 & 7.33 & 2.14 \\
\hline A5 & 2500 & 600 & 7.44 & 159 & 65 & 845 & 7.83 & 3.14 \\
\hline B1 & 1110 & 15 & 7.23 & 184 & 28 & 471 & 3.66 & 2.15 \\
\hline B2 & 1110 & 30 & 7.49 & 198 & 30 & 489 & 3.83 & 2.00 \\
\hline B3 & 1110 & 60 & 7.50 & 203 & 34 & 523 & 4.33 & 2.03 \\
\hline B4 & 1110 & 120 & 7.58 & 210 & 41 & 591 & 5.33 & 2.15 \\
\hline B5 & 1110 & 600 & 7.64 & 206 & 65 & 1194 & 11.00 & 2.57 \\
\hline
\end{tabular}

\section{b) Sonolyse of pretreated water}

In these experimental tests, raw water was treated with aluminum sulphate (coagulation process) and the liquid phase after decantation was used for sonolysis trials. Operating parameters and characterization data are presented in table 2. Most of the experiments were done to $1110 \mathrm{~W}$ energy of the ultrasonic wave (samples C1-C8), with variation of irradiation time $(15 \div 600 \mathrm{sec}$.) at different wave amplitude values $(25 \div 100 \%)$; the last two (C9-C10) tests were done to $555 \mathrm{~W}$ for maximum wave amplitude.

Table 2. Sonolyse experiments of pretreated water

\begin{tabular}{|c|c|c|c|c|c|c|c|c|c|}
\hline Sample & Energy & $\begin{array}{c}\text { Amplit. } \\
\%\end{array}$ & $\begin{array}{c}\text { Irrad. } \\
\text { time } \\
\text { sec. }\end{array}$ & $\mathrm{pH}$ & Turbidity & Temp. & Conductivity & COD & DOC \\
\hline $\begin{array}{c}\text { Pretreat } \\
\text { water }\end{array}$ & - & - & - & 6.9 & 7.2 & 25 & 514 & 5.64 & 2.68 \\
\hline C1 & 1110 & 100 & 15 & 6.91 & 9.5 & 28 & 533 & 2.50 & 2.65 \\
\hline C2 & 1110 & 100 & 30 & 6.70 & 9.3 & 29 & 536 & 1.56 & 2.27 \\
\hline C3 & 1110 & 100 & 60 & 6.64 & 8.8 & 32 & 576 & 3.76 & 2.42 \\
\hline C4 & 1110 & 100 & 120 & 6.63 & 9.7 & 38 & 646 & 3.13 & 2.79 \\
\hline C5 & 1110 & 100 & 600 & 7.47 & 14.5 & 68 & 989 & 3.13 & 2.69 \\
\hline C6 & 1110 & 50 & 15 & 6.65 & 11 & 32 & 576 & 2.98 & 2.67 \\
\hline C7 & 1110 & 25 & 30 & 6.59 & 10 & 29 & 537 & 2.82 & 2.13 \\
\hline C8 & 1110 & 50 & 60 & 6.84 & 12 & 49 & 772 & 3.45 & 2.56 \\
\hline C9 & 555 & 100 & 120 & 6.60 & 12.7 & 31 & 547 & 2.66 & 2.69 \\
\hline C10 & 555 & 100 & 600 & 6.81 & 11.6 & 39 & 647 & 3.13 & 2.35 \\
\hline
\end{tabular}


Characterization data of the treated samples emphasized the following main aspects:

- the turbidity rises with irradiation time for all values of energy and wave amplitude, maximum being 14.5 NTU (sample C5: irradiation 10 minutes, energy $1110 \mathrm{~W}$, amplitude 100\%);

- the temperature increases with time for all energies and amplitudes with a maximum value to $68{ }^{\circ} \mathrm{C}$ corresponding to sample $\mathrm{C} 5$;

- the conductivity rises slowly for short irradiation time and became accelerated over two minutes (4\% after $15 \mathrm{sec}$., $25 \%$ after two minutes and $53 \%$ after ten minutes) showing the dissolution of suspended matter;

- the degradation efficiency of organic matter expressed by DOC had variations as following:

- for the same values of energy and amplitude, sonolyse leads to DOC decreasing with $15 \%$ maximum after $30 \mathrm{sec}$. (sample C2);

- diminishing of wave amplitude from $100 \%$ to $25 \%$ at the same irradiation time in case of sample $\mathrm{C} 2$ leads to a higher mineralization efficiency - $20 \%$ (sample C7);

- the pairs of samples C4/C9 (sonolyse two minutes) and C5/C10 (sonolyse ten minutes) were compared; the results showed that it is possible to reach $12 \%$ efficiency of organic carbon mineralization for higher irradiation time and low wave energy $(555 \mathrm{~W})$; in these particular cases, operating conditions were favorable in terms of organic carbon balance - the amount of organic carbon which is mineralizing is higher than the amount of organic carbon which is dissolving from the suspended matter in the same time (sample C10);

- table 3 shows the values of nonconventional and auxiliary indicators which were selected for the characterization of pretreated water and treated samples (samples C4, C5, C6, C7, C8, C10). The data correlation between conventional indicators (table 2) and nonconventional/auxiliary indicators leads to some observation referring both to efficiency of pretreatment and sonolyse process and to the probability of THM generation during the disinfection because of residual natural organic matter.

Table 3. Sonolyse experiments of pretreated water - nonconventional indicators

\begin{tabular}{|l|c|c|c|c|c|c|c|}
\hline Nonconventional & Pretreated & \multicolumn{6}{|c|}{ Water samples after sonolyse } \\
\cline { 3 - 8 } indicators & water & C4 & C5 & C6 & C7 & C8 & C10 \\
\hline A254, $\mathrm{cm}^{-1}$ & 0.0268 & 0.0252 & 0.0305 & 0.0284 & 0.0256 & 0.0296 & 0.0291 \\
\hline A280, cm ${ }^{-1}$ & 0.0195 & 0.018 & 0.0225 & 0.0179 & 0.0187 & 0.0217 & 0.0205 \\
\hline A365, cm ${ }^{-1}$ & 0.0033 & 0.0027 & 0.0041 & 0.0022 & 0.0024 & 0.0042 & 0.0033 \\
\hline $\begin{array}{l}\text { SUVA } \\
\text { (A254/DOC), L }\end{array}$ & 1 & 0.9 & 1.1 & 1.03 & 1.2 & 1.1 & 1.2 \\
mg $^{-1} \mathrm{~m}^{-1}$ & & & & & & & \\
\hline A254/A280 & 1.37 & 1.4 & 1.35 & 1.59 & 1.37 & 1.36 & 1.42 \\
\hline A254/A365 & 8.1 & 9.3 & 6.1 & 12.9 & 10.7 & 7 & 8.8 \\
\hline
\end{tabular}


A large part of NOM constituents is hydrophobic and their oxidation (sonolyse generates hydroxyl radicals, one of the most powerful oxidants) leads to hydrophilic compounds soluble in water. Thus, partial oxidation of NOM aromatic compounds increases hydrophilic component and the absorbance to $\lambda$ $=254 \mathrm{~nm}$ will increase proportionally (A254 indicator).

In case of six selected samples (UV spectrum for each of them) it can be noted that almost A254 values are higher than initial sample (pretreated water).

The corresponding DOC concentrations are higher than initial value $(2.68 \mathrm{mg}$ $\mathrm{C} / \mathrm{l})$ excepting samples $\mathrm{C} 7$ and $\mathrm{C} 10$, which were performed in favorable conditions for organic carbon mineralization. Thereby, conventional indicator DOC can be correlated with A254 nonconventional indicator.

A280 indicator is useful to collect information about typical aromatic compounds able to generate THM compounds.

A365 indicator offers indications about the presence of aromatic and hydrophobic compounds. Small values indicate a good efficiency of coagulation-flocculation treatment step.

SUVA is commonly used indicator for comparison of aromatic feature of humic substances and as a measure of coagulation process efficiency.

SUVA values for pretreated and treated water are around 1 which means that pretreatment step (coagulation with aluminum sulphate) was efficient and the compounds are mainly hydrophilic.

High levels of SUVA $(>4)$ indicate predominance of aromatic hydrophobic species.

A254/A280 ratio offers information about the amount of NOM compounds with high potential to generate THM. Low level indicates the presence of these compounds which absorb to $\lambda=280 \mathrm{~nm}$.

Figures 1-2 show the variations of light absorbance ratio for four ultrasonic treated samples $(\mathrm{C} 4, \mathrm{C} 5, \mathrm{C} 6, \mathrm{C} 7)$ and for initial pretreated water.

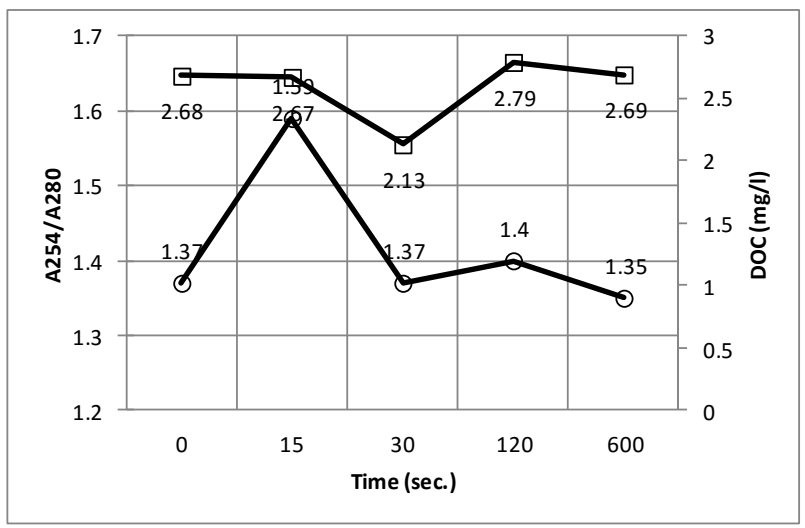

Figure 1 Variation of A254/A280 indicator and DOC with sonolyse time

A254/A280 is greater in case of samples with higher DOC values where the sonolyse was less efficient (15 sec. reaction time). After $30 \mathrm{sec}$. (lowest DOC concentration value) there is a continuous presence of compounds with THM potential generation. 


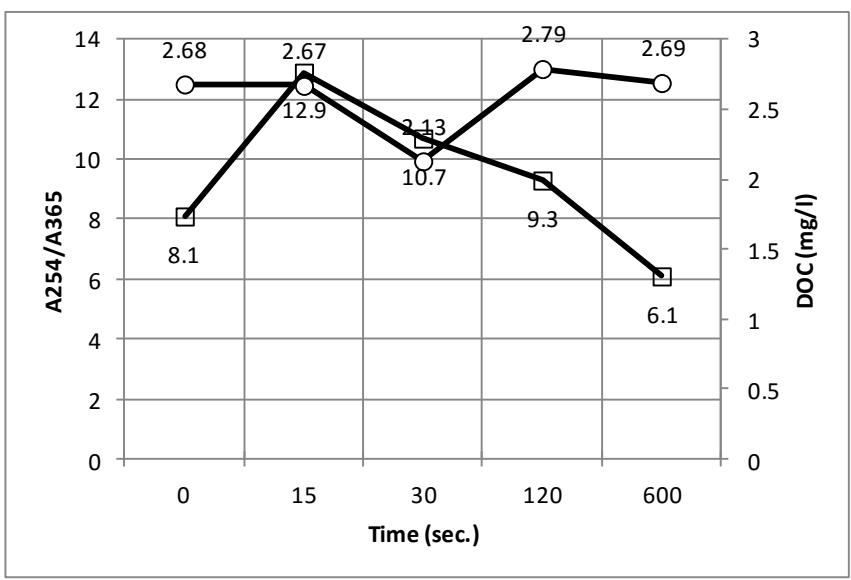

Figure 2 Variation of A254/A365 indicator and DOC with sonolyse time

High values of A254/A365 indicator (selected samples had A254/A365>6) emphasize the presence of aromatic compounds with high molecular weight which can be the result of humic substances degradation during the sonolyse water treatment. In these selected tests, 15-30 seconds of ultrasonic irradiation emphasized this aspect (high value of A254/A365 an lowest value of DOC in case of $\mathrm{C} 7$ sample - 30 sec. reaction time).

\section{Conclusions}

The experiments of drinking water (surface water) sonolyse were performed in order to establish optimal conditions (irradiation time, energy, amplitude, temperature) for natural organic matter degradation/removal taking into account that NOM are the main precursors of THMs, generated during final chlorine disinfection step.

The correlation of conventional water characterization indicators (COD, DOC, conductivity a.s.o.) with nonconventional indicators (A254, A280, A365, SUVA) and auxiliary indicators (A254/280, A254/365) leads to the following conclusions:

- direct sonolyse $(16 \mathrm{kHz})$ can remove organic load expressed by DOC conventional indicator with $15-20 \%$ efficiency; improving efficiency can be done by copling with other advanced oxidation;

- in the absence of temperature control, high NOM removal efficiencies were noted for short irradiation time (30 sec.), medium energy (1110 W) and low amplitude of the ultrasonic wave $(25 \%)$ or for longer irradiation time (time $\geq 10 \mathrm{~min}$.) but with low energy ultrasonic wave $(555 \mathrm{~W})$;

- nonconventional and auxiliary indicators emphasized that pottential of THM generation still remain after sonolyse (A254/A280 1) in the selected experimental conditions. 


\section{Acknowledgements}

This work was supported by a grant of the Romanian National Authority for Scientific Research - Programme Nucleu 13N/2009 -Project PN 09-13 0316

\section{References}

[1] Virkutyte J., Rokhina E.V., (2010), Hybrid Advanced Oxidation Techniques Based on Cavitation for Micropollutants Degradation, Treatment of Micropollutants in Water and Wastewater, Jurate Virkutyte, IWA Publishing, 321-353

[2] Mason T.J., Petrier C., (2004), Ultrasound Processes, Advanced Oxidation Processes for Water and Wastewater Treatment, Parsons Simon, IWA Publishing, UK, 185-206

[3] Cosma C., Nicolau M., Patroescu V., Stefanescu M., Ballo A., Florescu S., (2009), The incidence of By-products (THMs) Disinfection in Drinking Water, Journal of Environmental Protection and Ecology, 1, 14-22

[4] Culea M., lordache A., Ristoiu D., (2009), Trihalomethanes Analysis in Drinking Water, Journal of Environmental Protection and Ecology, 2, 342350

[5] Kochubovski M., (2009), Water Safety in Small-scale Supplies and New Approaches, Journal of Environmental Protection and Ecology, 12, 2017

[6] Masu S., (2011), Simultaneous Reduction of Turbidity and Natural Organic Mater by Simple and Prehydrolized Aluminium Salts, Revista de Chimie, 62, 1, 64-68

[7] Masu S., Albulescu M., Turuga L., (2010), Natural Organic Matter from Surface Water:Potential Risk for Drinking Water, Annals of West University of Timisoara, 19, 3, 9-16 